\section{Centre of attention}

Polycomb group ( $\mathrm{PcG}$ ) proteins stably repress gene expression and form complexes with each other. PcG complexes, in turn, form discrete nuclear foci, known as PcG bodies, into which other transcriptional regulators might be recruited. As reported in Cell, the PcG protein Pc2 has now been identified as a new SUMO E3 that recruits the transcriptional corepressor $\mathrm{CtBP}$ and the $\mathrm{E} 2$ (conjugating) enzyme Ubc9 to PcG bodies, thereby enhancing CtBP sumoylation.

The single sumoylation motif in CtBP hinted to David Wotton's group that CtBP could be sumoylated. In vitro, CtBP sumoylation minimally required recombinant $\mathrm{E} 1$ (an activating enzyme), Ubc9 and SUMO-1. In vivo, CtBP immunoprecipitated from HeLa cells was detected with an anti-SUMO-1-specific antibody. Lysine 428 of CtBP was identified as the principal site for SUMO conjugation, and the surrounding motif was important for efficient sumoylation.

\section{SENESCENCE}

\section{A challenging overhang}

The replicative lifespan of cells is thought to be determined by one of two mechanisms that ultimately trigger senescence cumulative DNA damage or the progressive shortening of telomeres. Robert Weinberg and colleagues, reporting in Nature Genetics, now challenge the 'telomere length' hypothesis.

Weinberg and colleagues examined the possible role of the $3^{\prime}$ telomeric overhang a key component of the telomere structure that is composed of several hundred nucleotides of the G-rich strand extended beyond the end of the $\mathrm{C}$-rich strand during senescence. For this, they used a newly developed method, the telomereoligonucleotide ligation assay (T-OLA), in which radiolabelled oligonucleotides complementary to the overhang are annealed, and then ligated, to genomic DNA. The length and intensity of the ligation products, as measured by electrophoretic

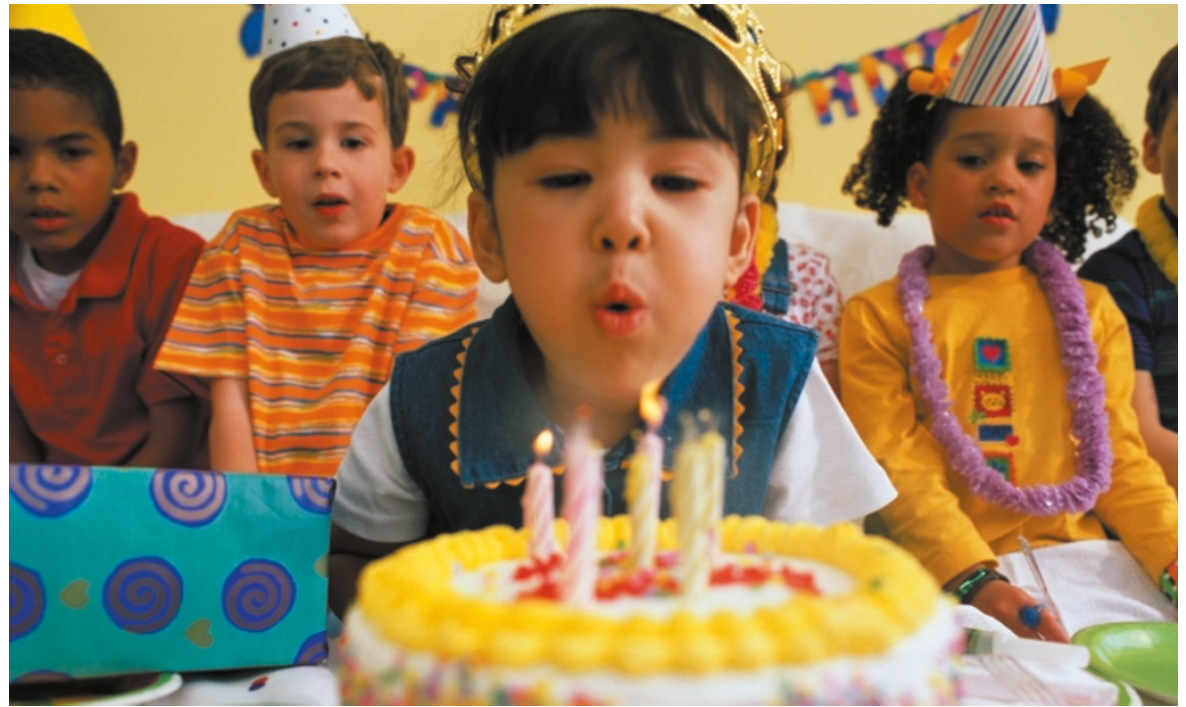

$\mathrm{CtBP}$ can be nuclear and cytoplasmic but, in the presence of recombinant Pc2, much of it colocalized with Pc2 in nuclear foci, where it was found to be sumoylated. Pc2 itself was also sumoylated, so could Pc2 affect SUMO localization? Co-expressing Pc2 caused SUMO-1 and Ubc9 to move to nuclear foci. Pc2 colocalized there, too, suggesting that these foci were probably PcG bodies.

As Pc2 co-expression caused the recruitment of a SUMO substrate (CtBP) and an E2 enzyme (Ubc9) to the same compartment, the authors tested Pc2's ability to enhance CtBP sumoylation. In cell-based and in vitro sumoylation assays, recombinant Pc2 increased the amount of sumoylated CtBP

So, PcG bodies might function as centres of sumoylation in the nucleus. The authors speculate that "...PcG complexes may exert their effects on transcription in part by mediating the sumoylation of specific target proteins" and that CtBP, which might bridge interactions between transcriptional regulators, along with Pc2, could be "...part of the PcG sumoylation machine...".

(4) References and links

Katrin Bussell

ORIGAL RESEARCH PAPER Kagey, M. H., Melhuish, T. A. \& Wotton, D. The Polycomb protein Pc2 is a SUMO E3. Cell 113, 127-137 (2003) separation and densitometric analysis, respectively, indicate the length of the overhangs present in the sample.

Using T-OLA, the authors measured the telomeric overhang in BJ fibroblast cell lines. By population doubling 106, when virtually all BJ cells were senescent, the maximal detected length was 384 nucleotides, compared with 624 nucleotides in earlypassage cells, and the overall signal intensity had decreased to $19 \%$ of that in early-passage cells. Similar results were found in a second fibroblast cell line.

Expression of telomerase is known to prevent senescence, which Weinberg and colleagues explain by the enzyme's ability to extend the $3^{\prime}$ overhang. So is the overhang loss the cause or the consequence of senescence? To test this, the authors examined whether the telomeric overhang was lost in cells that avoid senescence by inactivation of $\mathrm{p} 53$ and retinoblastomaassociated protein (RB). Overhang loss in these cells was similar to that in senescent cells, indicating that it was a result of continuous cell division rather than senescence itself. In addition, the authors found no evidence that the quiescent state of senescent cells or physiological stress are responsible for the extent of telomeric

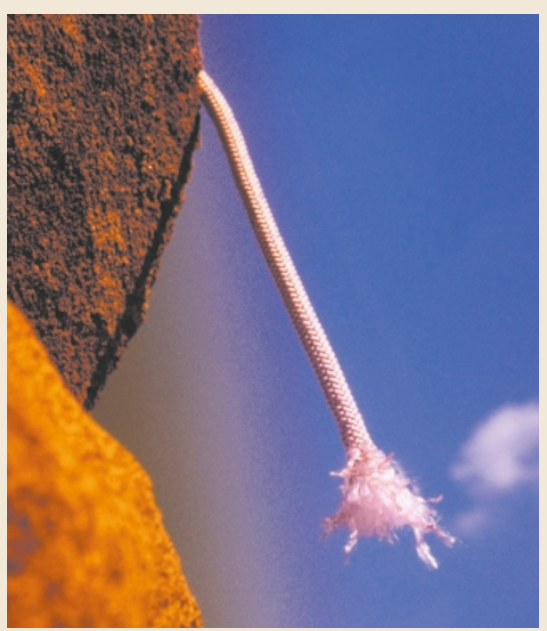

overhang loss seen in senescent cells.

So, the authors conclude that "...overhang loss, rather than shortening of overall telomere length, is the molecular signal that triggers senescence". They propose that the overhang loss might affect a crucial telomeric structure, such as the T-loop.

Arianne Heinrichs

(2) References and links

ORIGINAL RESEARCH PAPER Stewart, S. A. et al. Erosion of the telomeric single-strand overhang at replicative senescence. Nature Genet. 2003 March 24 (DOI: 10.1038/ng1127) 